\title{
Odonto-onycho dysplasia-alopecia syndrome
}

INSERM

\section{Source}

INSERM. (1999). Orphanet: an online rare disease and orphan drug data base. Odontoonycho dysplasia-alopecia syndrome. ORPHA:2722

Odonto-onycho dysplasia-alopecia syndrome is a rare, genetic ectodermal dysplasia syndrome characterized by almost total alopecia with only sparse, thin, brittle, slowgrowing scalp hair, fair and sparse eyebrows and eyelashes, absent axillary and pubic hair, fragile and brittle fing ernails, thick and brittle toenails (both with a subungual corneal layer), hypodontia, microdontia, widely spaced teeth with hypoplastic enamel, mild palmoplantar keratosis, café-au-lait spots and areolae anomalies. There have been no further descriptions in the literature since 1985. 